\title{
Implicit and explicit Motivated Self-Perception as hypothesis-driven self-construal
}

\author{
Leah M. Kaufmann ${ }^{1 *}$ \\ Victor E. Sojo ${ }^{2}$ \\ Anna E. Genat ${ }^{3,4}$ \\ Melissa A. Wheeler ${ }^{3}$ \\ Robert E. Wood ${ }^{3,5}$ \\ ${ }^{1}$ School of Psychology, Australian Catholic University \\ ${ }^{2}$ Centre for Workplace Leadership, The University of Melbourne \\ ${ }^{3}$ Centre for Ethical Leadership, Ormond College, The University of Melbourne \\ ${ }^{4}$ Melbourne Business School, The University of Melbourne \\ ${ }^{5}$ Australian Graduate School of Management, University of New South Wales
}

*Correspondence to: Dr Leah Kaufmann

School of Psychology

Australian Catholic University

Locked Bag 4115 Fitzroy MDC

Fitzroy, VIC 3065 Australia

The authors declare no potential conflicts of interest with respect to the research, authorship, and/or publication of this article. This research did not receive any specific grant from funding agencies in the public, commercial, or not-for-profit sectors. 
- Motivated Self-Perception affects the explicit, remembered, and implicit self.

- Motivated Self-Perception involves evidencing an explicitly desired self-construal.

- Self-construal creates a desired implicit self from a hypothesized explicit self.

- Motivated Self-Perception reflects explicit and implicit representations of traits. 


\title{
Implicit and explicit Motivated Self-Perception as hypothesis-driven self-construal
}

\begin{abstract}
Motivated Self-Perception (MSP) facilitates the positive perception of the self via the endorsement of desirable characteristics, selective recall of autobiographical memories, and performance of desirable behaviour. Peters and Gawronski (2011) proposed a model of MSP as "hypothesis-driven" self-construal integrating implicit and explicit self-concepts, motivation, and autobiographical memory. The current study provides the first complete test of this model. One hundred and twenty-seven participants read a summary of a fictional study before completing measures of motivation, personality self-ratings, autobiographical memory, and implicit self-personality associations. Explicit self-concept, autobiographical memory, and the implicit self-personality association were affected by the manipulation, consistent with predictions. Results also revealed that implicit self-personality associations were predicted by motivation and autobiographical memory, providing evidence for the proposed model of MSP, and further evidence for the interconnectedness of implicit and explicit self-constructs. Finally, these results are interpreted as evidence for the effect of MSP-based self-enhancement across all levels of the self.
\end{abstract}

Key words: motivated self-perception, implicit self-concept, hypothesis-driven selfconstrual, personality, autobiographical memory 
Abbreviations:

MSP Motivated Self-Perception

I Introversion

E Extraversion

MSPI Motivated Self-Perception - introversion (condition)

MSPE Motivated Self-Perception - extraversion (condition)

GNAT Go/No Go Association Task

ANOVA Analysis of Variance 


\section{Introduction}

Motivated Self-Perception (MSP; Sanitioso, Kunda, \& Fong, 1990) is the perception of socially desirable attributes as true of one's self (e.g., Kunda, 1987). MSP has been found to affect self-ratings (Kunda \& Sanitioso, 1989), autobiographical memory (e.g., Brunot \& Sanitioso, 2004), desirable behaviour (e.g., Augustinova, Collange, Sanitioso, \& Musca, 2011), and even implicit self associations (Peters \& Gawronski, 2011). MSP research has demonstrated that inducing the desirability of a characteristic can result in the perception of this characteristic as being part of the self at explicit, remembered, and implicit levels.

Peters and Gawronski’s (2011) hypothesis-driven self-construal integrates theory and research providing an explanation of the interconnection of implicit and explicit self-concepts and autobiographical memory, and provides a complete model of MSP effects across all levels of the self. However, the complete model remains untested. The current study will test this model by enhancing the desirability of Extraversion (E) or Introversion (I) in a yokedcontrol design (Church, 1964) to examine explicit, autobiographical memory, and implicit MSP effects. In doing so, we consider the impact of the inherently differentially desirable factor of E (e.g., Duffy \& Chartrand, 2015), and motivation from induced desirability of E versus I, on self-perceptions across all levels of the self.

\subsection{MSP review}

The typical MSP paradigm was developed by Kunda and Sanitioso (1989) who had student participants read that Extraversion (E) or Introversion (I) was associated with academic success and attempt to explain the finding. The students then completed a purportedly unrelated measure of personality and demonstrated higher self-ratings for the desirable trait (e.g., E or I, respectively). This finding was consistent with previous research that found that participants who were informed that more or less frequent action was associated with health resulted in a corresponding increase or decreased the reported 
frequency of the action (e.g., more frequent tooth-brushing - Ross, McFarland, \& Fletcher, 1981; consumption of caffeine - Sherman \& Kunda, cited in Kruglanski, 1996).

Explicit (i.e., self-reported) MSP occurs only if the manipulation enhances the desirability of the attribute and is personally relevant. For example, Sanitioso and colleagues' (1990) found that participants who were induced to believe a trait was conducive to the success for police officers (i.e., a profession of little interest to student participants) were unaffected by the MSP manipulation, though they rated the trait as desirable. This supports the MSP interpretation over that of socially desirable responding (e.g., Paulhus \& John, 1998) as desirability was independent of self-ratings and ineffective alone. In addition, MSP for E and I have been found to be constrained by participants' own E and I, assessed previously. This finding suggests that MSP self-ratings reflect participants' personality rather than the desirability of the trait.

Sanitioso and colleagues (1990) proposed that explicit MSP effects reflect a biased search of autobiographical memory facilitating the perception of a desirable trait as being true of self. Four distinct findings support this argument. First, participants typically reported significantly more desirable trait-relevant autobiographical memories (Sanitioso et al., 1990). Second, desirable trait-relevant autobiographical memories were recalled with greater perceived ease than undesirable trait-relevant autobiographical memories (Sanitioso \& Niedenthal, 2006). Third, desirable trait-relevant autobiographical memories were recalled more in general or typical terms, rather than in specific or instance-based terms (Brunot \& Sanitioso, 2004). Finally, desirable trait-relevant autobiographical memories were recalled more from a first, compared to a third, person perspective (Sanitioso, 2008). These findings show that MSP affects both content and experience of autobiographical memory making the desirable attribute appear typical and internally, rather situationally attributable (e.g., Kelley, 1973). 
Beyond self-perception, Sanitioso and Wlodarski (2004) found participants preferred partners who confirm MSP-consistent self-perception. Furthermore, Augustinova and colleagues (2011) found that participants who were induced to believe rationality (versus intuition) was associated with success perceived themselves as more rational and demonstrated greater use of base-rate sensitivity than availability heuristics to solve reasoning problems (Kahneman \& Tversky, 1973). These participants also engaged in more time-consuming or effortful consideration of a problem, and provided more accurate solutions. In sum, participants who believed that rationality was associated with success saw themselves as more rational, behaved more rationally, and were more successful than those who did not. These findings are strong evidence for (at least) temporarily motivated change in the self, which can be perceived by self and others via behaviour.

\subsection{Implicit MSP}

Peters and Gawronski (2011) proposed an interconnected and mutually influencing relationship between implicit and explicit self-concepts via two processes of self-construal. First, a bottom-up process allows the explicit self to be construed from information in autobiographical memory and validated by implicit self-associations. That is, a person answering the question "who am I?" introspects on their behaviour and experiences, activating context-relevant autobiographical memories and implicit self associations, resulting in a "data-driven" or bottom-up self-construal. This process facilitates consistency across self-aspects and results in substantial consistency across (particularly similar) situations. In contrast, the context-consistent dynamic self results from the "hypothesisdriven” or top-down process of self-construal. Specifically, motivation leads to a confirmatory search of autobiographical memory and biased activation of motivationconsistent implicit self associations to validate this explicit proposition (e.g., "I am 
extraverted"). Importantly, this process of self-construal describes both the mechanism of MSP, and proposes a model for MSP-based change across all levels of the self.

Support for Peters and Gawronski's (2011) processes or self-construal were demonstrated by two findings. First, the significant relationship between autobiographical recall and explicit self-rated personality was fully mediated by implicit self-personality associations, supporting for their proposed bottom-up process (Study 1). In Study 2, the typical MSP induction resulted in stronger implicit associations between self-desirable trait and other-undesirable trait, compared to self-undesirable trait and other-desirable trait using Implicit Association Test (IAT; Greenwald, McGhee, \& Schwarz, 1998). Moreover, implicit self associations were predicted by the induction condition, and this relationship was fully mediated by explicit personality self-ratings consistent with the hypothesis-drive process, and was the first demonstration of implicit MSP.

\subsection{The current study}

The current study was designed to conceptually replicate implicit MSP, and to extends this by testing Peters and Gawronski's (2011) proposed model of MSP (i.e., hypothesisdriven self-construal). Specifically, the effects of an MSP induction will be measured (i.e., motivation), and its effect on implicit and explicit self-concepts, and autobiographical memory will be assessed. Finally, the contribution of each of these variables to the prediction of the implicit self-concept will be examined. In doing so, we will provide the first test of the Peters and Gawronski's model of MSP. Furthermore, by using the Go/No Go Association Task (GNAT; Nosek \& Banaji, 2001) in place of the IAT (Greenwald et al., 1998), we will eliminate the confound of inseparable self and other implicit associations (i.e., IAT scores reflect both targets and attribute, whereas each GNAT $d$ ' reflects only one target and attribute; see Williams \& Kaufmann, 2012). This allows exploration of the distinct effects of MSP on 
self-I and self-E implicit associations which will provide further insight into the nature of how this personality factor functions explicitly and implicitly.

Consistent with previous MSP findings, we propose that:

1. Participants who were informed that I was associated with success (MSPI condition) will endorse I as more related to success than those who were informed that $\mathrm{E}$ has been found to be associated with success (MSPE condition). This will provide a measure of motivation or trait desirability.

2. Participants in the MSPI condition will endorse I as more true of self than MSPE participants.

3. Participants will recall a higher percentage of memories related to the successrelated trait.

4. Participants will demonstrate stronger implicit associations between the self and the success-related trait.

5. Enhanced perception of the I- or E-success relationship via MSP will predict self-I and self-E implicit associations via motivation and autobiographical memory (see Figure 2) consistent with Peters and Gawronski’s (2011) proposed model.

\section{Method}

\subsection{Participants}

Participants were 127 undergraduate psychology students including 18 males (14.3\%) and one participant who did not indicate their gender. Participants ranged from 18 to 58 years $(M=22.06, S D=5.43)$. The majority $(86.6 \%)$ of participants were born in Australia, and all met the English language university entry requirements. Three (1.9\%) participants were excluded from analyses for incomplete data and 27 (18.0\%) for poor performance on the GNAT. 


\subsection{Materials and measures}

MSP induction. Participants read a summary of a fictional study which was designed to manipulate the desirability of I (MSPI) or E (MSPE). The summaries were 98 words linking I or E to academic and professional success. The only difference between the summaries was a sentence attributing the cause of success to independence (i.e., MSPI) or “networking” (i.e., MSPE; see Supplement).

Motivation. The effectiveness of the MSP manipulation was assessed by participant ratings of the advantageousness of I to academic success (i.e., "How advantageous is Introversion to academic success") and professional success, to health, and in general on a 10-point scale, from 1 (not at all advantageous) to 10 (highly advantageous). The degree to which participants associated I with each type of success was used as an index of induced motivation.

Personality ratings. Participants rated themselves on a single item using a scale from 1 (Introverted) to 10 (Extraverted).

Autobiographical memory task. Participants reported memories of personal events or experiences which demonstrate the dimension "shy" (i.e., I) or "outgoing" (E; e.g., Sanitioso et al., 1990). Each memory was coded either as I (e.g., "missed out on going with others because I was not comfortable") or E (e.g., "threw a party"). Any responses that were not clearly relevant (e.g., "was extravagant”) were excluded from analysis. Responses were independently coded by two of the authors and tallied as a function of I memories $(r=.95)$ or E memories ( $r=.94)$.

GNAT. Implicit associations between self and I and self and E were assessed by the GNAT (Nosek \& Banaji, 2001). Self, other, I, and E were represented by six stimuli (e.g., “SELF”, “OTHER”, “RESERVED”, “SOCIABLE”, respectively; see Supplement) and were 
used as targets and distracters. For example, for the block where self and I were targets, other and E stimuli were used as distracters.

Blocks comprised 10 practice trials and 70 experimental trials with equal numbers of target and distracter trials. Targets were presented prior to the start of each block that block. Trials terminated when a response was made or after $750 \mathrm{~ms}$. Participants received feedback at the end of each trial (a red " $\mathrm{X}$ " for incorrect responses or a green "O" for correct responses presented for $350 \mathrm{~ms}$ ), followed by $300 \mathrm{~ms}$ inter-trial interval. A short break was suggested after each block.

The self-I and self-E implicit associations were calculated using the equal variance $d^{\prime}$ formula (e.g., Wickens, 2002) with target trials treated as signal (i.e., coded as hits or misses) and distracter trials treated as noise (i.e., coded as false alarms or correct rejections). Higher values indicate stronger implicit associations (Nosek \& Banaji, 2001).

\subsection{Design and Procedure}

Participation was undertaken in laboratory classes in an undergraduate psychology unit. Participants read a plain language statement and indicated their informed consent before completing any measures. Students accessed the online experiment hosted at www.millisecond.com which randomly assigned each participant to the between-subjects conditions of MSPI and MSPE. All participants completed the motivation induction first, followed by the manipulation check, self-ratings, autobiographical memory, and implicit association measures. A measure of accessibility (e.g., a lexical decision task) was conducted, but is not presented as it does not form part of the proposed model of MSP. Student participants were offered the opportunity to withdraw their data prior to screening which was taken by four participants $(2.6 \%)$. 


\section{Results}

\subsection{Descriptive statistics and correlations by MSP condition}

Participants in the MSPE condition produced only two means that were higher than participants in the MSPI condition. Specifically, participants in the MSPE condition produced more E-related memories and implicitly associated self more with E than participants in the MSPI condition (See Table 1).

Correlations between variables as a function of MSP condition (MSPI above the diagonal; MPSE below the diagonal) are presented in Table 1. The measures of the I advantageousness were all positively correlated, most significantly so. The pattern of correlations between memory measures was consistent across conditions, the only difference being that some correlations failed to reach significance in the MSPE condition. Finally, the implicit associations between self and I and E were correlated in both MSP conditions.

Table 1 about here

\subsection{MSP components}

Consistent with Hypothesis 1, participants in the MSPI rated I as significantly more advantageous to professional and academic success than participants in the MSPE condition. Importantly, no significant difference in perceived advantageousness of I was observed for the scales of health and in general, which were not explicitly described in the experimental manipulation (see Table 2).

Table 2 about here 
Consistent with Hypothesis 2, participants in the MSPI condition rated their personality as marginally more I than participants in the MSPE condition (see Table 2). Consistent with Hypothesis 3, participants in the MSPI condition reported a higher percentage of I-related memories than those in the MSPE condition (see Table 2).

Hypothesis 4 was explored as the effect of MSP on implicit self associations using a mixed-model ANOVA. Significant main effects were found for the within-subjects factor of implicit personality (implicit I, E associations) with all participants demonstrating stronger self-I than self-E implicit associations $F(1,123)=14.63, p<.01, \eta_{\mathrm{p}}{ }^{2}=.11$. However, this effect was qualified by an interaction with the between-subjects factor of MSP condition $F(1,123)=5.33, p=.02, \eta_{\mathrm{p}}{ }^{2}=.04$. Participants in the MSPE condition demonstrated self-I $(M=2.33, S E=.12,95 \% \mathrm{CI}=2.08,2.57)$ and self-E $(M=2.19, S E=.12,95 \% \quad \mathrm{CI}=1.96,2.42)$ implicit associations of similar magnitudes (Hedge's $g=.14$ ). In contrast, in the MSPI condition participants demonstrated a stronger self-I $(M=2.53, S E=0.15,95 \% \mathrm{CI}=2.24,2.81)$ implicit associations than a self-E $(M=1.98, S E=0.14,95 \% \mathrm{CI}=1.71,2.5)$ implicit association (Hedge's $g=.49$ ) as can be seen in Figure 1.

Figure 1 about here

\subsection{Testing the self-construal model of MSP}

To test Hypothesis 5, whether the effect of MSP on the self-I and self-E implicit associations is mediated by motivational factors and the salience of the memories that the manipulation elicits, two parallel mediation models using Hayes' (2013) PROCESS macro (Model 4) with 10,000 bias corrected bootstrap samples were run. MSP condition (I=1, E=0) was the main predictor, the two motivation factors (level of endorsement of 
advantageousness of I to professional and to academic success) and the percentage of I memories were used as mediators and the implicit measures of I and E were used as dependent variables. All variables except MSP condition were $z$-transformed. Figure 2 illustrates the conceptual model.

Figure 2 about here

There were two significant mediators of the effect of MSP conditions on implicit selfI association (see Table 3a), namely, the percentage of I memories (indirect effect=.13, $S E=.07[95 \%$ CI .04, .29]) and the evaluation of I as advantageous to professional success (indirect effect=.10, SE=.06 [95\%CI .02, .23]). Comparison of indirect effects revealed only the indirect effect of the percentage of I memories was significantly different from the indirect effect of evaluation of I as advantageous to academic success (contrast $=-.14$, $S E=.09,[95 \% \mathrm{CI}-.32,-.02])$.

Only the percentage of I memories mediated the association between MSP conditions and the implicit self-E association (see Table 3b; indirect effect=.12, SE=.07 [95\% CI $\left.0.04,0.27]^{1}\right)$. When we compared the indirect effects, only the indirect effect of the percentage of I memories was significantly different from the path of evaluation of I as advantageous to professional success (contrast $=-.18, S E=.09,[95 \% \mathrm{CI}-.35,-.05]$ ), the other contrasts were not significant.

Tables $3 \mathrm{a}$ and $3 \mathrm{~b}$ about here

\footnotetext{
${ }^{1}$ We also tested an alternative model with Self-I and Self-E implicit associations as mediators of the relationship between MSP manipulation and the percentage of I memories and found no mediation effect, consistent with Peters and Gawronski (2010) findings.
} 


\section{Discussion}

Our data provides the first complete test of Peters and Gawronski's (2011) model of MSP, and reveals that MSP operates across all levels of the self. Specifically, we found that (H1) participants in the MSPI condition rated I as significantly more advantageous to academic and professional success; (H2) rated themselves as marginally higher on I; (H3) reported a significantly higher percentage of I memories; and (H4) demonstrated higher self-I implicit associations and lower self-E implicit associations compared to MSPE participants. Finally, parallel mediation analysis revealed that (H5) self-I and self-E implicit associations were accounted for by models comprising MSP condition, motivation, and autobiographical memory. In sum, moderate to strong support was found for all hypotheses.

Examination of the parallel mediation models revealed different effects for I and $\mathrm{E}$ implicit associations, as well as for the components of the MSP model, although this may be affected by the order of tasks. Specifically, only autobiographical memories predicted both self-I and self-E implicit associations. Whereas, motivation was found to be a significant predictor of self-I implicit associations, and MSP condition was a significant predictor of self-E implicit associations. These findings likely reflect the inherent differences between I and E (e.g., E is more socially desirable; Duffy \& Chartrand, 2015) resulting in the MSPI manipulation enhancing only the less desirable trait.

Our results provide further support for implicit MSP, but differ in important ways from Peters and Gawronski’s (2011) findings. First, our study tested (and supported) Peters and Gawronski's untested model of MSP. Second, Peters and Gawronski predicted a single implicit self outcome calculated as a difference score (i.e., self-I and other-E was subtracted from self-E and other-I). In contrast, we examined the effects of MSP on self-I and self-E implicit associations separately, allowing the MSP effect for each to be examined and 
avoiding problems arising from the use of difference scores (see Williams \& Kaufmann, 2012).

The current research addressed several weaknesses of previous studies. First, we addressed the uninterpretable "other" resulting from the use the IAT. Second, we examined all proposed components affecting I- and E-self implicit associations from MSP separately, providing support for the proposed model of MSP (i.e., hypothesis-driven self-construal), and allowed identification of differences between implicit and explicit personality not previously addressed. Specifically, while there is a bias towards the endorsement of E consistent with the differential desirability of I and E (e.g., Duffy \& Chartrand, 2015), reverse pattern is found for implicit associations (i.e., self-I>self-E).

\section{Asymmetric Implicit and Explicit Personality}

The differences in I and $\mathrm{E}$ in implicit and explicit personality can be easily interpreted through models of implicit and explicit constructs (e.g., Associative Propositional Evaluation model; Gawronski \& Bodenhausen, 2006). Specifically, explicit constructs are propositional, acquired quickly and deliberately (e.g., "I am outgoing and sociable") whereas implicit constructs are associational, acquired slowly and unintentionally (e.g., each experience contributes to E or I self associations). Consider, for example, a time when you sang in public which is likely to both be recalled long after the event, and inform your answer to the question "how outgoing am I?" In contrast, the implicit system accumulates daily experiences of E (e.g., a single public speaking engagement) and I (e.g., many quiet nights at home) in the proportion that they typically occur resulting in a tendency towards stronger self-I than self-E implicit associations, consistent with the current findings.

Despite differences in the levels of implicit and explicit I and E, the current findings suggest MSP functions consistently across the self, with the most effective manipulation occurring for typically less desirable or frequent characteristics. Consequently, high explicit 
and low implicit levels of E are observed, and there is limited flexibility to self-enhance on E. In contrast, I is less desirable but extremely common and can be explicitly and implicitly enhanced, as observed in the current findings.

\section{Future Directions and Conclusions}

Two avenues of research arise from the current findings. First, our findings suggest that MSP has the potential to affect all levels of the self in motivation-consistent ways. That is, motivation aligns self with a desired outcome, shaping who we are and even how we behave (e.g., Augustinova et al., 2011). Such a phenomenon could be a powerful tool for selfimprovement, especially if MSP enhanced self in ways consistent with the desired outcome, and if such changes persist. Implicit theories research (e.g., Dweck 2006) demonstrates the usefulness of adaptive mindsets which can be induced, and which have lasting real-world consequences (e.g., Yeager \& Dweck, 2012). However, the potential for MSP to yield similar positive outcomes has not been explored. This is a limitation of the current research and typical of MSP research generally.

For future research to examine the positive consequences of MSP, several changes to the typical method would be required. First, MSP typically uses between-subject rather than within-individual changes; therefore, it is not currently correct to interpret MSP as change. Research using a control or fully repeated design is required to facilitate this conclusion. Second, examination of the duration of MSP effects is warranted. Given the typical stability of implicit constructs once established (e.g., Gregg, Seibt, \& Banaji, 2006), MSP may have a more sustained effect than has been posited (Sanitioso et al, 1990). Moreover, if enduring personal changes were intended, ethical consideration mandates the use of factual or at least beneficial manipulations. In addressing these shortcomings, the applications of MSP for positive self-change or therapy may be revealed (e.g., Teachman \& Woody, 2003). 
A final contribution of the current research, and avenue for future work, is the integration of implicit and explicit theory into personality research. To date, research on implicit personality has focused on measurement (e.g., validity and reliability; Banse \& Greenwald, 2007), or exploration of correlations between implicit and explicit measures of factors (e.g., Big Five personality traits - Grumm \& von Collani, 2007), often ignoring known theoretical differences between implicit and explicit constructs. Our research progresses knowledge in this area by grounding implicit and explicit constructs of personality in theory, and demonstrating meaningful differences between implicit and explicit representations of I and E. In doing so, we have taken a first essential step to integrating these literatures. 


\section{REFERENCES}

Augustinova, M., Collange, J., Sanitioso, R. B., \& Musca, S. C. (2011). Power of the desired self: Influence of induced perceptions of self on reasoning. Cognition, 121, 299-312. doi:10.1016/j.cognition.2011.08.004.

Brunot, S., \& Sanitioso, R. B. (2004). Motivated influence on the quality of memories: Recall of general autobiographical memories related to desired attributes. European Journal of Social Psychology, 34, 627-635. doi:10.1002/ejsp.220

Church, R. M. (1964). Systematic effect of random error in the yoked control design. Psychological Bulletin, 62, 122-131. doi:10.1037/h0042733

Duffy, K. A., \& Chartrand, T. L. (2015). The extravert advantage: How and when extraverts build rapport with other people. Psychological Science, 26, 1795-1802. doi:10.1177/0956797615600890

Dweck, C. S., (2006). Mindset. New York, NY: Random House.

Fazio, R. H., \& Olson, M. A. (2003). Implicit measures in social cognition research: Their meaning and use. Annual Review of Psychology, 54, 297-327. doi:10.1146/annurev.psych.54.101601.145225

Gawronski, B., \& Bodenhausen, G. V. (2006). Associative and propositional processes in evaluation: An integrative review of implicit and explicit attitude change. Psychological Bulletin, 132, 692-731. doi:10.1037/0033-2909.132.5.692

Gregg, A. P., Seibt, B., \& Banaji, M. R. (2006). Easier done than undone: asymmetry in the malleability of implicit preferences. Journal of Personality and Social Psychology, 90, 1-20. doi:10.1037/0022-3514.90.1.1

Greenwald, A. G., McGhee, D. E., \& Schwartz, J. K. (1998). Measuring individual differences in implicit cognition: The implicit association test. Journal of Personality and Social Psychology, 74, 1464-1480. doi:10.1037/0022-3514.74.6.1464 
Kahneman, D., \& Tversky, A. (1973). On the psychology of prediction. Psychological Review, 80, 207-232. doi:10.1016/0010-0285(73)90033-9

Kruglanski, A. W. (1996). Motivated social cognition: Principles of the interface. In E. T. Higgins and A. W. Kruglanski (Eds). Social psychology: Handbook of basic principles (pp 493-520). New York, NY: Guilford Press.

Kunda, Z. (1987). Motivation and inference: Self-serving generation and evaluation of evidence. Journal of Personality and Social Psychology, 53, 636-647. doi:10.1037/0022-3514.53.4.636

Kunda, Z., \& Sanitioso, R.B. (1989). Motivated changes in the self-concept. Journal of Experimental Social Psychology, 25, 272-285. doi:10.1016/0022-1031(89)90023-1

Nosek, B. A., \& Banaji, M. R. (2001). The go/no go association task. Social Cognition, 19, 625-666. doi:10.1521/soco.19.6.625.20886

Paulhus, D. L., \& John, O. P. (1998). Egoistic and moralistic biases in self-perception: the interplay of self-deceptive styles with basic traits and motives. Journal of Personality, 66, 1025-1060. doi:10.1111/1467-6494.00041

Peters, K. R., \& Gawronski, B. (2011). Mutual influences between the implicit and explicit self-concepts: The role of memory activation and motivated reasoning. Journal of Experimental Social Psychology, 47, 436-442. doi:10.1016/j.jesp.2010.11.015

Ross, M., McFarland, C., \& Fletcher, G. J. (1981). The effect of attitude on the recall of personal histories. Journal of Personality and Social Psychology, 40, 627-634. doi:10.1037/0022-3514.40.4.627

Sanitioso, R. B. (2008). Motivated self and recall: Visual perspectives in remembering past behavior. European Journal of Social Psychology, 38, 566-575. doi:10.1002/ejsp.456 
Sanitioso, R. B., Kunda, Z., \& Fong, G. T. (1990). Motivated recruitment of autobiographical memories. Journal of Personality and Social Psychology, 59, 229-241. doi:10.1037/0022-3514.59.2.229

Sanitioso, R. B., \& Niedenthal, P. M. (2006). Ease of recall and motivated self-perception. Self and Identity, 5, 73-84. doi:10.1080/15298860500386848

Sanitioso, R. B., \& Wlodarski, R. (2004). In search of information that confirms a desired self-perception: Motivated processing of social feedback and choice of social interactions. Personality and Social Psychology Bulletin, 30, 412-422, doi:10.1177/0146167203261882

Schnabel, K., Banse, R., \& Asendorpf, J. B. (2006). Assessment of implicit personality self-concept using the implicit association test (IAT): Concurrent assessment of anxiousness and angriness. British Journal of Social Psychology, 45, 373-396. doi:10.1348/014466605X49159

Teachman, B. A., \& Woody, S. R. (2003). Automatic processing in spider phobia: Implicit fear associations over the course of treatment. Journal of Abnormal Psychology, 112, 100-109. doi:10.1037/0021-843X.112.1.100

Wickens, T. D. (2002). Elementary Signal Detection Theory. NY: Oxford University Press. Williams, B. J., \& Kaufmann, L. M. (2012). Reliability of the Go/No Go Association Task. Journal of Experimental Social Psychology, 48, 879-891. doi:10.1016/j.jesp.2012.03.001

Yaeger, D. S., \& Dweck, C. S. (2012). Mindsets that promote resilience: When students believe that personal characteristics can be developed. Educational Psychologist, 47, 302-314. doi: 10.1080/00461520.2012.722805 
Table 1 Summary of Correlations, Means (and SDs) for Motivation, Measures of Explicit, Remembered, and Implicit Self as a Function of MSP Condition

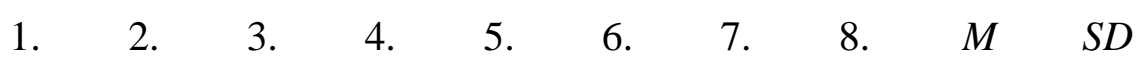

Advantageousness of I

1. to health

2. in general

3. to professional success

4. to academic

$.36^{* *} .34^{* *} \quad .19$

Success

5. Personality self$<.01 \quad-.04 \quad-.02 \quad-.17$ $\begin{array}{llll}.54^{* *} & .05 & .07 & 5.31\end{array}$ rating

6. Autobiographical $\begin{array}{llll}.09 & -.07 & -.02 & -. .10\end{array}$ .20 memory $(\% \mathrm{I})$

Implicit associations

\begin{tabular}{|c|c|c|c|c|c|c|c|c|c|}
\hline 7. Self-I & .17 & .18 & .22 & -.13 & -.05 & $.26^{*}$ & & $.55^{* *}$ & 2.53 \\
\hline 8. Self-E & -.03 & -.01 & -.05 & -.04 & .08 & .23 & $.52^{* *}$ & & 1.95 \\
\hline$M$ & 6.17 & 5.99 & 5.08 & 5.90 & 4.68 & 24.17 & 2.33 & 2.19 & \\
\hline$S D$ & 1.88 & 1.79 & 2.68 & 1.75 & 1.69 & 26.34 & 1.02 & .87 & \\
\hline
\end{tabular}

Note. Correlations for MSPI condition are above the diagonal $(n=52-55)$; correlations for MSPE are below ( $n=71-72)$.

Personality self-rating and personality ratings of memories: 1=outgoing, 10=shy. First memory recalled: $0=\mathrm{E}, 1=\mathrm{I}$. 
Table 2

Comparison of Dependent Variables as a Function of MSP Condition

$\begin{array}{llll}t & d f & \text { Hedges' }\end{array}$

\begin{tabular}{lcccc} 
& & & & $g$ \\
\hline Advantageousness of I & -3.86 & 125 & $<.01$ & -.66 \\
Professional success & -3.26 & 125 & $<.01$ & -.58 \\
Academic success & -1.21 & 125 & .23 & -.21 \\
General & -0.43 & 125 & .67 & -.73 \\
Health & -1.80 & 125 & .07 & -.32 \\
Personality self-rating & & & & \\
Autobiographical memory & & & & \\
\% I memories & -2.81 & 124 & .01 & -.50 \\
\hline
\end{tabular}

Note. Negative $t$ values indicate MSPI>MSPE. Higher ratings of personality rating reflects higher levels of I. 
Table 3a

Parallel Mediation Model for the Prediction of Self-I Implicit Associations

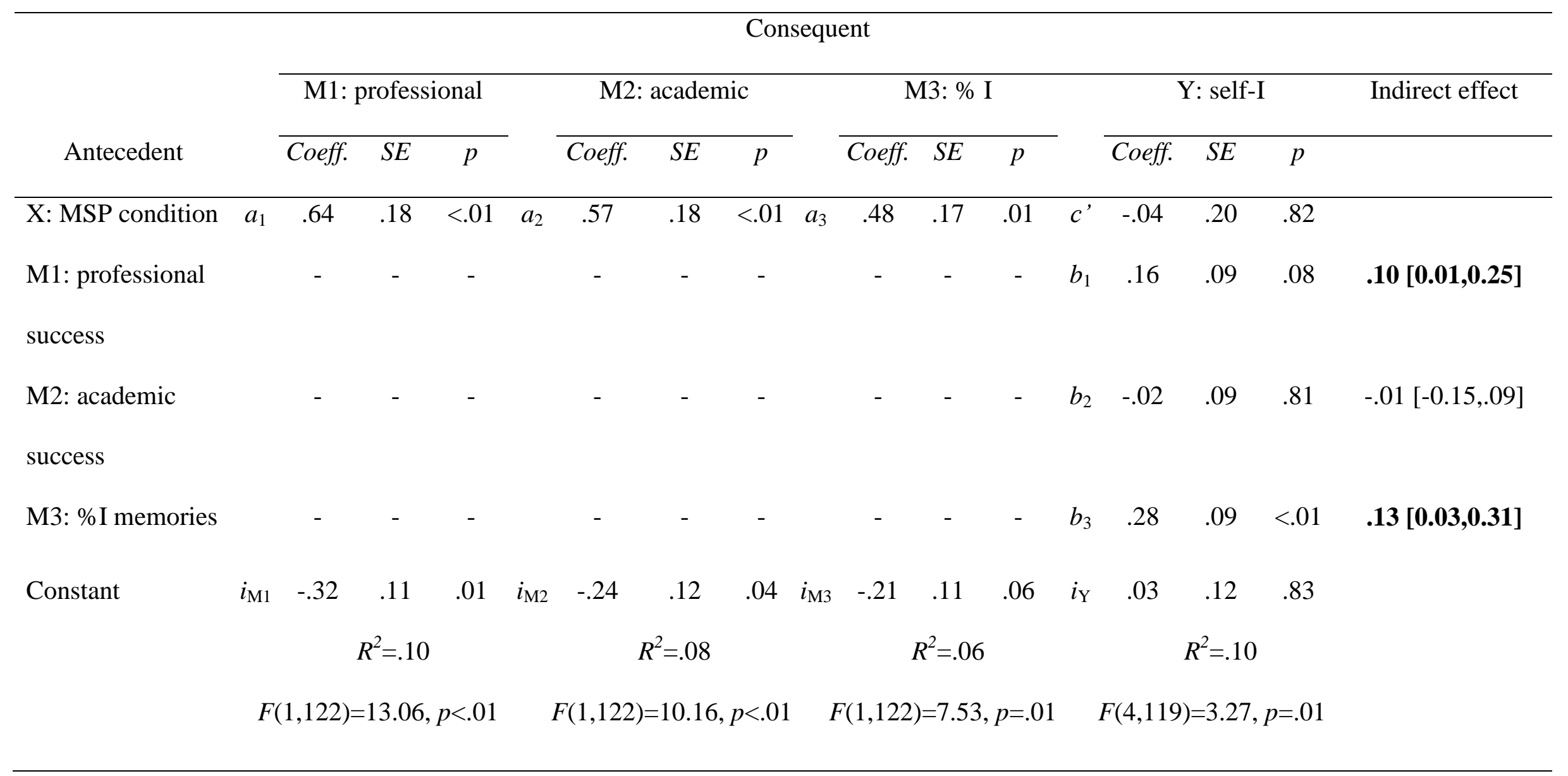


Table $3 b$

Parallel Mediation Model for the Prediction of Self-E Implicit Associations

\begin{tabular}{|c|c|c|c|c|c|c|c|c|c|c|c|c|c|c|c|c|c|}
\hline \multirow[b]{3}{*}{ Antecedent } & & \multicolumn{15}{|c|}{ Consequent } & \multirow{3}{*}{ Indirect effect } \\
\hline & \multicolumn{4}{|c|}{ M1: professional } & & \multicolumn{3}{|c|}{ M2: academic } & \multicolumn{4}{|c|}{ M3: \% I } & & \multicolumn{3}{|c|}{ Y: self-E } & \\
\hline & & Coeff. & $S E$ & $p$ & & Coeff. & $S E$ & $p$ & & Coeff. & $S E$ & $p$ & & Coeff. & $S E$ & $p$ & \\
\hline $\mathrm{X}$ : MSP condition & $a_{1}$ & .65 & .17 & $<.01$ & $a_{2}$ & .60 & .18 & $<.01$ & $a_{3}$ & .49 & .18 & .01 & $c^{\prime}$ & -.34 & .20 & .08 & \\
\hline M1: professional & & - & - & - & & - & - & - & & - & - & - & $b_{1}$ & -.08 & .09 & .40 & $-.05[-0.19,0.06]$ \\
\hline \multicolumn{18}{|l|}{ success } \\
\hline M2: academic & & - & - & - & & - & - & - & & - & - & - & $b_{2}$ & .04 & .09 & .68 & $.02[-0.09,0.13]$ \\
\hline \multicolumn{18}{|l|}{ success } \\
\hline M3: \% I memories & & - & - & - & & - & - & - & & - & - & - & $b_{3}$ & .25 & .09 & .01 & $.12[0.03,0.32]$ \\
\hline \multirow[t]{3}{*}{ Constant } & $i_{\mathrm{M} 1}$ & -.32 & .11 & .01 & $i_{\mathrm{M} 2}$ & -.24 & .12 & .04 & $i_{\mathrm{M} 3}$ & -.21 & .11 & .07 & $i_{\mathrm{Y}}$ & .14 & .12 & .24 & \\
\hline & & \multicolumn{4}{|c|}{$R^{2}=.10$} & \multicolumn{3}{|c|}{$R^{2}=.08$} & \multicolumn{5}{|c|}{$R^{2}=.06$} & \multicolumn{3}{|c|}{$R^{2}=.08$} & \\
\hline & \multicolumn{5}{|c|}{$F(1,124)=14.06, p<.01$} & \multicolumn{3}{|c|}{$F(1,124)=11.31, p<.01$} & \multicolumn{4}{|c|}{$F(1,124)=7.87, p=.01$} & & \multicolumn{3}{|c|}{$F(4,121)=2.69, p=.03$} & \\
\hline
\end{tabular}




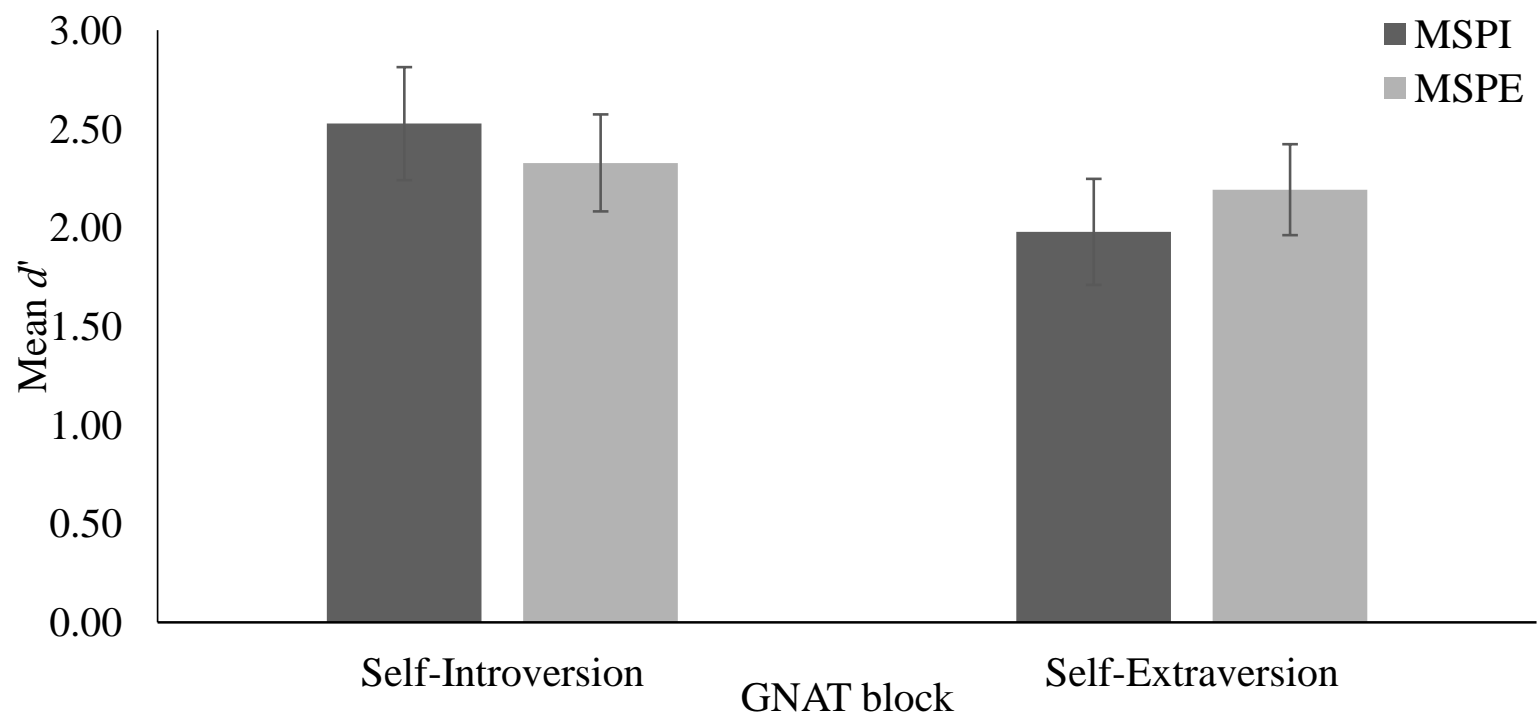

Figure 1. Mean $d^{\prime}$ for implicit self associations as a function of MSP condition. Error bars represent $95 \% \mathrm{CI}$.

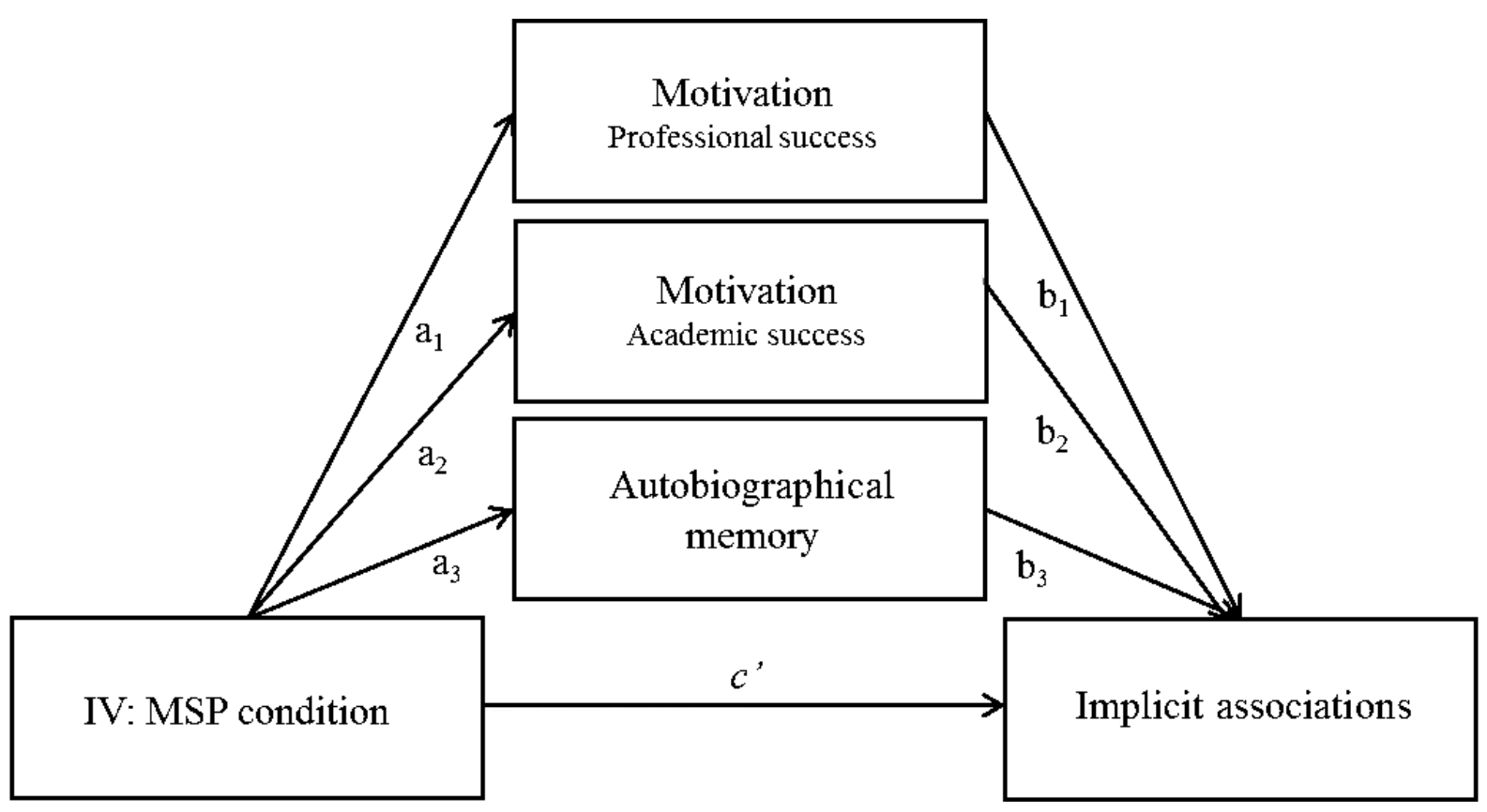

Figure 2. Theoretical parallel mediation model - motivation vs explicit memory as mediator. 


\section{SUPPLEMENT - Stimuli}

\section{Manipulation stimuli}

\section{Introversion success condition}

A recent study examining factors affecting health and wellbeing among Australians aged 21 to 55 years found that people who were introverted were typically better paid than their extraverted counterparts. Higher levels of introversion were associated with spending more time independently leading to personal and professional achievements which has been hypothesised to be responsible for greater work-life balance and higher levels of professional success. Finally, introversion has been linked to greater resilience to negative events and more effective coping styles thought to help people handle the pressure of day-to-day work.

Journal of Australian Organisational Psychology, vol 11, 2014

\section{Extraversion success condition}

A recent study examining factors affecting health and wellbeing among Australians aged 21 to 55 years found that people who were extraverted were typically better paid than their introverted counterparts. Higher levels of extraversion were associated with spending more networking and socialising helping these people "get ahead" which has been hypothesised to be responsible for greater work-life balance and higher levels of professional success. Finally, extraversion has been linked to greater resilience to negative events and more effective coping styles thought to help people handle the pressure of day-to-day work. Journal of Australian Organisational Psychology, vol 11, 2014 


\section{GNAT stimuli}

Self: SELF, ME, MINE, I, OWN, AM

Other: OTHER, THEY, THEM, YOU, THEIR, GROUP

Extraversion: OUTGOING, CONFIDENT, TALKATIVE, SOCIABLE, FRIENDLY, BRASH

Introversion: QUIET, RESERVED, INDEPENDENT, BOOKISH, RESTRAINED, SHY 